\title{
Musculosceletal tuberculosis with involvement of tendon sheaths and formation of synovial cyst
}

The authors declare no finacial disclosure

\begin{abstract}
Due to an increasing amount of patients on immunosuppressive treatment, the number of tuberculosis (TB) of atypical course and extrapulmonary tuberculosis cases increase. Locomotor system is a place of every fifth case of extrapulmonary TB. Because of lack of characteristic symptoms, as well as rare co-occurrence of active lung lesions in radiological imaging, proper diagnosis is hard to establish. We present a case of patient on immunosuppressive therapy due to myositis, in whom we diagnosed musculoskeletal tuberculosis in form of involvement of tendon sheath and formation of synovial cyst.
\end{abstract}

Key words: extrapulmonary tuberculosis, ganglion cyst, tendon sheath inflammation

Pneumonol Alergol Pol 2016; 84: 278-282

\section{Introduction}

Active tuberculosis (TB) remains an important public health problem and one of the leading causes of death of infectious diseases in the world. The analysis of TB in the area of the United States during the years 1993-2006 have shown that the total number of TB cases has decreased, however the number of cases of extrapulmonary tuberculosis is increasing relative to the forms of pulmonary TB. It was responsible for every fifth case of tuberculosis in 2006. In 2002, tuberculosis was the 10th cause of disability in the world. Due to increasing amount of patients with acquired immunodeficiency and on immunosuppressive treatment of other diseases, the number of tuberculosis of atypical course increases [1, 2]. In Poland, in 2015 a total of 6430 cases of tuberculosis were registered. Of this number, 352 cases (which accounted for $5.5 \%$ of all registered cases) were extrapulmonary tuberculosis and patients without lung involvement. Musculoskeletal system was involved in 39 cases, and location of lesions in spine were reported in 16 people [3].
Musculoskeletal involvement usually develops on the basis of reactivation of primary foci of the disease and blood-borne dissemination. It can develop due to the primary infection in the motor organ caused by direct inoculation, e.g during operation, by using surgical instrument contaminated with mycobacteria, though it is rare. Mycobacteria settle mainly in areas with extensive vascular network, in which blood flow is slowed down. In the musculoskeletal system it corresponds to the long bone epiphysis and the vertebral body. The inflammatory process leads to the destruction of soft tissue and bone structures, suggesting rheumatologic background and delays the proper diagnosis [4]. Obtaining a positive result of bacteriological or histological examination is the basis for correct diagnosis. The material may be obtained by biopsy or, in more advanced cases, as a result of surgical resection of the lesions [4, 5].

We present a case of patient on glucocorticoid therapy due to myositis. The patient was suspected of multiple myeloma. Periarticular

Address for correspondence: Michał Zieliński, Department of Lung Diseases and Tuberculosis, School of Medicine with the Division of Dentistry in Zabrze,

Medical University of Silesia in Katowice, e-mail: michal.zielinski1@interia.pl

DOI: 10.5603/PiAP.2016.0035

Received: 15.05.2016

Copyright (C) 2016 PTChP

ISSN 0867-7077 
lesions were diagnosed as a manifestation of tuberculosis after fine needle biopsy and surgical biopsy of the changes.

\section{Case report}

79-year-old woman was admitted to the Department of Lung Diseases because of the presence of nodular changes of soft tissues surrounding the left wrist joint and detection of tuberculous granulomas and Mycobacterium tuberculosis complex DNA in the biopsy sample from the changes.

The first respiratory symptom was the presence of bilateral pleural effusion found in the chest radiograph taken during hospitalization in Urological Department due to urinary tract infection and left-sided nephrolithiasis in March 2013. In the next chest radiograph, taken one week after the last one, during another hospitalization because of a myocardial infarction with a left bundle branch block, formerly seen bilateral pleural effusion and streak-like shadows were detected.

In the control chest radiograph right-sided pneumothorax with the level of fluid was revealed. It was qualified for conservative treatment. Due to the increasing value of TSH, the patient was transferred to the Endocrinology Department to compensate the endocrine system. The radiological imaging performed during this hospitalization (April 2013) showed resorption of pneumothorax, and persistence of pleural effusion, mainly on the right side. Thoracentesis was not performed because of too small amount of effusion.

Due to the presence of nodular changes in the soft tissue surrounding the left wrist, and the pain in the joints of both hands, in August 2013, ultrasound of the area of both wrists and hands was performed. Inflammatory lesions and overload changes of extensor tendons, with limited mobility and the presence of sclerosing fluid in peritendon were revealed. Because of these changes and indurations in the muscles of both forearms, prednisone at a dose of $20 \mathrm{mg}$ daily was prescribed for suspected myositis.

The patient was hospitalized again at the turn of September and October 2013 in Neurology and Internal Diseases Departments because of increasing shoulder and pelvic girdle muscle weakness. Again, chest radiograph revealed pleural effusion, however in smaller amounts as compared to previous studies. In addition (to that do usunięcia), interstitial changes in lower parts of lungs were revealed. Thoracentesis was not performed again because of too small amount of effusion. CT-scan of the chest revealed compression of the basal and posterior lung fields by the effusion. Radiological findings were consistent with the presence of inflammatory changes in the compressed lung tissue, with suspicion of secondary mediastinal lymphadenopathy. Emphysematous lesions in the upper and the middle lobes were also described.

Electromiography (EMG) examination detected features of myogenic muscle damage, which address polymyositis. The presence of serum IgG/kappa monoclonal protein and Anti-Sjögren's-syndrome-related antigen A (SSA) autoantibodies were detected. The patient was offered a muscle biopsy to confirm the diagnosis of polymyositis, however she did not consent. The obtained bone marrow aspiration biopsy suggested multiple myeloma.

Because of the bone marrow biopsy diagnosis, the hematologic diagnostics was performed at the Department of Hematology and Transplantation in January and February 2014. There were no osteolytic lesions in the comprehensive skeletal imaging. The results of the other tests did not allow for confirmation of multiple myeloma diagnosis and monoclonal gammapathy of undetermined significance IgG kappa was diagnosed instead. In May 2014, the next ultrasound of wrists revealed effusion and hypertrophy of synovial sheaths in the surrounding of the left hand tendons. Outlines of joints were smooth and the findings characteristic of degenerative changes were not revealed. In October 2014 the patient undertook orthopedic surgery consultation. Consultant of orthopedic surgery described multiple soft tissue tumors and subcutaneous tissue infiltration in the upper limbs and suggested obtaining material from those lesions for histological examination. In the material from the area on the left shoulder, left shoulder joint ganglion cyst was macroscopically described. Microscopic examination revealed TB-like granulomas with necrosis in the central part.

The patient was referred to the outpatient pulmonary department, in which, in November 2014 material for further diagnostics from changes in the left wrist was taken (Fig. 1). In genetic examination, presence of DNA of Mycobacterium tuberculosis complex was revealed. Moreover positive culture on Lowenstein-Jensen medium was obtained. The patient was referred to the Department of Pneumology. In the time of admission, the patient was treated with: $2.5 \mathrm{mg}$ of bisoprolol, $15 \mathrm{mg}$ of prednisone, $500 \mathrm{mg}$ of sulfasalazine, $20 \mathrm{mg}$ of pantoprazole, probiotic with Saccharomyces boulardii, $75 \mathrm{mg}$ of aspirin, 0,25 ug of alfakalcydiol, substitution with levothy- 


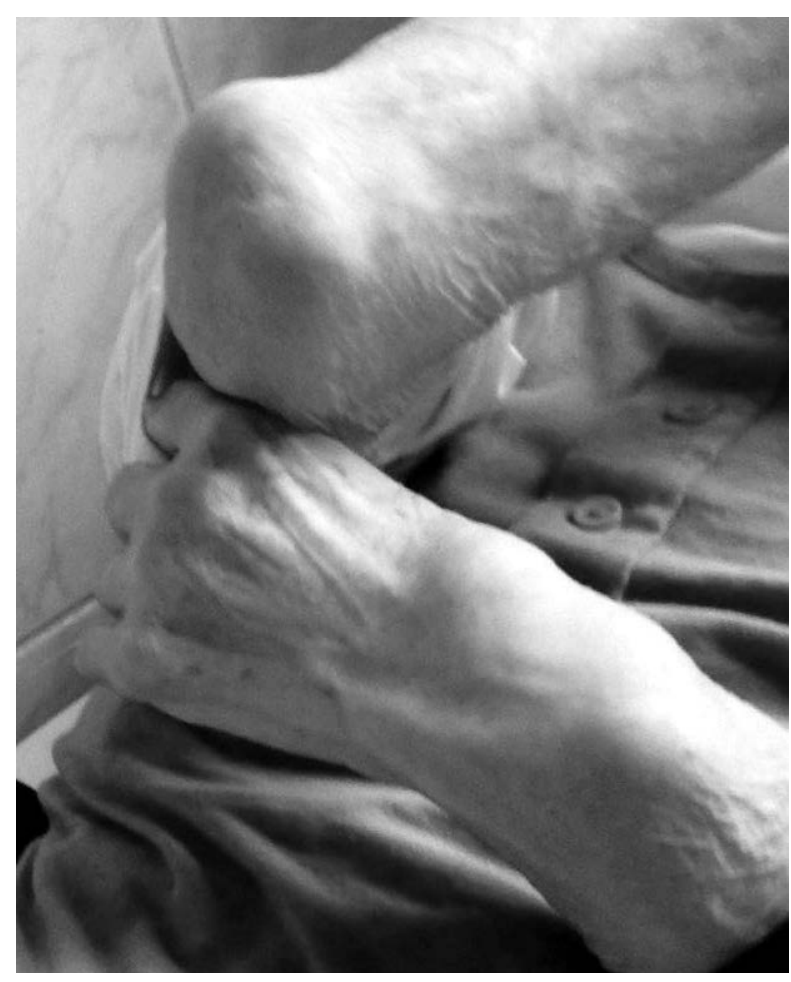

Figure 1. Pseudonodular lesions in the area of the right elbow and left wrist - the place from material was taken

roxine (175 ug for 5 days a week and 150 ug for 2 days in the week). In addition to that, the patient underwent a cataract surgery on both sides, cholecystectomy, and radioiodine treatment of hyperthyroidism. In 1978, she suffered from hepatitis B. She was under constant control of nephrology and cardiology outpatient departments due to chronic kidney disease, mixed hyperlipidemia, and heart failure. The patient denied any contact with people affected with tuberculosis

Except elevated CRP levels to $15 \mathrm{mg} / \mathrm{L}$ and elevated serum creatinine to $136 \mathrm{mmol} / \mathrm{L}$, laboratory examinations (markers of liver function, kidney function tests, electrolytes, whole blood count and markers of inflammation) were within normal limits. Chest radiograph did not show any abnormalities. The high resolution computed tomography (HRCT) revealed lung nodules based on a broncho-vascular bunch in upper lung segments, with greater intensity in the right lung and right $6^{\text {th }}$ segment. Furthermore, three bullous lesions in lower lungs segments up to $18 \mathrm{~mm}$ of diameter and single calcification in the right $5^{\text {th }}$ segment was revealed (Fig. 2).

Radiological image did not suggest activity of the lung lesions. Sputum for mycobacteria culture was not performed. Further examinations did not revealed presence of pleural effusion. Due to the comorbid diseases and lack of thoracenteses during



Figure 2. Lung HRCT, nodular consolidations leaned on a vascular and bronchial bunch in upper lung segments

previous hospitalizations, unequivocal differential diagnosis of pleural lesions was not possible.

We prescribed rifampicin, isoniazid, pyrazinamide and ethambutol, observing an improvement within two weeks. The patient was referred to the pulmonary and tuberculosis outpatient department for further treatment. The patient was also referred to rheumatology outpatient department to make a decision about continuation of prednisone treatment.

\section{Discussion}

Tuberculosis involving locomotor system accounts for about $20 \%$ of tuberculosis worldwide. The dominant location, representing $50 \%$ of the cases is tuberculosis of the spine. Subsequently, most frequently, large joints such as the hip and knee are involved. Involvement of the upper limb, as in our patient, accounts for $10 \%$ of all changes in bones. Articular changes in the course of tuberculosis begins with swelling of the soft tissue with bone-sparing, and then reaches the progressive destruction with the bone erosion of the articular surfaces, the formation of bone cysts, subluxations in the joints, and finally, ankylosis of the joint. Based on changes in the course of tuberculosis of the bone and joint, Tuli et al. proposed the classification of lesions into 5 stages. In our patient, radiological examinations did not reveal arthropathy, the ultrasound did not visualise features of destruction, and only involvement of soft tissues were described. According to the classification, such lesions may be allocated to the first stage. In this stage, proper treatment (anti-tuberculosis drugs, possibly immobilization and rehabilitation) promise a full recovery without leaving permanent consequences (Table 1) [6-9]. 
Tabela 1. Classification of changes in the course of tuberculosis of the joints, based on: [8]

\begin{tabular}{|c|c|c|}
\hline Stage & Clinical symptoms & Radiological changes \\
\hline I & $\begin{array}{l}\text { 1. Soft tissue swelling } \\
\text { 2. }>75 \% \text { motion range }\end{array}$ & $\begin{array}{l}\text { 1. Soft tissue swelling } \\
\text { 2. Osteopenia }\end{array}$ \\
\hline ॥ & $\begin{array}{l}\text { 1. Soft tissue swelling } \\
\text { 2. } 25-50 \% \text { of motion range }\end{array}$ & $\begin{array}{l}\text { Like in I, and additionally: } \\
\text { 1. The beginning of the erosion of the articular surfaces } \\
\text { 2. Moderate narrowing of the joint space }\end{array}$ \\
\hline III & 1. $25 \%$ of motion range & $\begin{array}{l}\text { Like in II, and additionally: } \\
\text { 1. A significant erosion of the articular surfaces } \\
\text { 2. Significant narrowing of joint space }\end{array}$ \\
\hline IV & $\begin{array}{l}\text { 1. }<25 \% \text { of motion range } \\
\text { 2. Subluxation or dislocation }\end{array}$ & $\begin{array}{l}\text { Like in III, and additionally: } \\
\text { 1. The destruction of joint with subluxation, dislocation }\end{array}$ \\
\hline V & 1. Ankylosis & $\begin{array}{l}\text { Like in IV, and additionally: } \\
1 . \text { Ankylosis }\end{array}$ \\
\hline
\end{tabular}

The diagnosis of tuberculosis in patients, who do not report respiratory symptoms, is a challenge. The general symptoms are uncharacteristic. Pain, swelling, joint stiffness, increase of the circumference of fingers, carpal tunnel syndrome and, in advanced form, cutaneous fistulas are the symptoms associated with the involvement of the joints. Because the clinical picture suggests rheumatic and orthopedic causes, diagnosis is delayed, which may result in a worse prognosis $[6,10,11]$. In patients reported in the studies of Turkish researchers, the most common symptoms were swelling and pain in joints, and a time to make the correct diagnosis reached 17 months. Notably, none of the described patients revealed changes in the chest radiograph that may correspond to active tuberculous changes in the lungs, which is consistent with the observation that the involvement of the locomotor system usually does not coexist with radiological changes suggestive of active TB lesions in chest $[6,11]$.

There is a possibility of the development of primary tuberculosis of the bone and joint caused by direct inoculation of the soft tissues of the joint. A case of a surgeon, who sustained a needlestick injury during the drainage of a perispinal abscess in a patient with disseminated tuberculosis was described. He developed swelling in his left hand and wrist, which did not respond to standard broad-spectrum antibiotic therapy. After anti-tuberculosis treatment, the symptoms withdrew [12]. In such cases, the clinical history clearly indicates the contact with potentially infectious material.

Diagnosis of extrapulmonary tuberculosis require positive culture growth or detection of tuberculous granulomas in histological examination of the affected organ. In the case of the musculoskeletal system, recommended materials are bone scrapings, the contents of a cold abscess, and synovial fluid [13]. During obtaining surgical material, in patients with soft tissue involvement of the joints, especially the hands, so-called "rice bodies" were reported - clusters of fibrous tissue and caseous necrosis in sheath tendons, which may lead to its rupture, as well as in the synovial membrane of joints, as a symptom of changes in synovial villi. They may occur in other diseases of the musculoskeletal system soft structures, however compared to those seen in tuberculosis they are smaller. Ganglion cyst, abscess, inflammation of the bursae and inflammation of the tendon sheaths encourage surgical intervention and obtaining the diagnostic material through surgery [6].

Fine needle aspiration (FNA) biopsy is a method that should be considered in the diagnostic process of extrapulmonary tuberculosis because of its simplicity, low invasiveness and cost-effectiveness. Ziehl-Neelsen stained material, obtained using this method, allows for visualization of acid-fast bacilli in $43.5 \%$ of cases. Acid-fast bacilli detection correlates with the presence of necrosis in the cytopathology examination [14]. In the studies, which analyzed the usefulness of the FNA biopsy and PCR methods in the diagnosis of extrapulmonary tuberculosis lymphadenopathy, high utility of these methods has been proved. The sensitivity was $90.3 \%$ and specificity was reported to reach $81.8 \%$. An additional advantage of the method is the short time from specimen collection to obtaining a result, which is several hours [15]. Similar results (sensitivity $87.8 \%$, specificity $91.1 \%$ ) were obtained for the system GeneXpert MTB/RIF, which in 2014 has been recommended by the WHO for the analysis of material from FNA in suspected extrapulmonary tuberculosis cases [16]. 
Apart from the Mycobacterium tuberculosis complex, extrapulmonary tuberculosis lesions in the soft tissue, can be caused by rapidly growing Mycobacterium abscessus. In addition to the respiratory infection, it most frequently causes changes in the skin, soft tissues, and bones. It has been reported in patients during chronic immunosuppressive therapy due to connective tissue disorders and transplant recipients with articular changes $[17,18]$. In presented patient, etiological factor of articular lesions was human Mycobacterium, however due to the immunosuppressive treatment and clinically significant drug resistance among atypical Mycobacteria, this etiology should be taken into account in the differential diagnosis.

In our patient, steroid therapy was prescribed because of suspected polymyositis. A number of disorders of the immune system is associated with susceptibility to tuberculosis. In the analysis involving two English databases covering about 50 million people observed in the years 1999 - 2011 and $1963-1998$, it was demonstrated that there is a significantly increased risk of tuberculosis in patients hospitalized for diseases associated with the dysfunction of immune system. Polymyositis and dermatomyositis were in the top ten of the analyzed diseases (rate ratio 8.0 and 6.6, respectively), while the risk of pulmonary and extrapulmonary tuberculosis for both diseases was comparable [19]. A similar analysis of the data from the Finnish register, covering the period from 1969 to 1985, showed that $10 \%$ of patients with polymyositis and dermatomyositis had an established diagnosis of tuberculosis in the period before the diagnosis of myositis. In addition, the cumulative probability of developing active TB within 5 years after the diagnosis of myositis was $4.8 \%$, rising to $9.3 \%$ after 10 years [20]. These associations are explained by a dysfunction of the immune system resulting from the underlying disease and immunosuppressive drugs used in the treatment. Presence of a mechanism that influences the susceptibility to both autoimmune diseases and tuberculosis is postulated [19, 21].

Tuberculosis is a disease which should be considered in the differentiation of articular changes. The increasing use of immunosuppressive therapy, including the new classes of drugs such as tumor necrosis factor alpha ( $\mathrm{TNF} \alpha$ ) antagonists, forces to search actively for changes that might be a source of TB prior to treatment and monitoring of the patient for the development of TB during treatment.

\section{Conflict of interest}

The authors declare no conflict of interest.

\section{References:}

1. Peto HM, Pratt RH, Harrington TA, LoBue PA, Armstrong LR. Epidemiology of extrapulmonary tuberculosis in the United States, 1993-2006. Clin Infect Dis 2009; 49: 1350-1357. doi: 10.1086/605559.

2. Mathers CD, Loncar D. Projections of global mortality and burden of disease from 2002 to 2030. PLoS Med 2006; 3: e442.

3. Gruźlica w Polsce w 2015 roku. Korzeniewska-Kosela M, Instytut Gruźlicy i Chorób Płuc, Warszawa 2016.

4. Malawski S. Gruźlica kości i stawów. W: Krakówka P., Rowińska-Zakrzewska E. (red.). Gruźlica. PZWL, Warszawa 1988: 151-167.

5. Shrestha OP, Spiegel DA, Banskota AK, Hosalkar HA. Bone and joint tuberculosis. UPOJ 2010; 20: 23-28.

6. Al-Qattan MM, Al-Namla A, Al-Thunayan A, Al-Omawi M. Tuberculosis of the hand. Hand Surg Am 2011; 36: 1413-1421.

7. Martin M, Benkeddache Y, Medjani Y, Gottesman H. Tuberculosis of the upper limb joints. Int Orthop 1986; 10: 17-23.

8. Spiegel DA, Singh GK, Banskota AK. Tuberculosis of the musculoskeletal system. Tech Orthop 2005; 20: 167-178.

9. Tuli SM. Principles of management of osteoarticular tuberculosis. W: Tuberculosis of the skeletal system: bones, hoints, spine and bursal sheaths, $5^{\text {th }}$ ed. Jaypee Brothers, Bangalore 2004; $56-67$.

10. Visuthikosol V, Kruavit A, Nitiyanant P, Siriwongpairat P. Tuberculous infection of the hand and wrist. Ann Plast Surg 1996; 37: 55-59

11. Subusi M, Bukte Y, Kapakaya A, Gurkan F. Tuberculosis of the metacarpals and phalanges of the hand. Ann Plast Surg 2004; 53: 469-472.

12. Lipani F, Canta F, Carosella S et al. Primary soft tissue and tenosynovial tuberculosis after needlestick injury in a surgeon. Infez Med 2008; 16: 33-36

13. Storm M., Vlok G.J. Musculoskeletal and spinal tuberculosis in adults and children. W: Schaaf H.S., Zumla A.I. (red.). Tuberculosis a comprehensive clinical reference. Saunders, Europe 2009; 494-503.

14. Narang S, Solanki A, Kashyap S, Rani L. Utility of fine needle aspiration cytology to comprehend the pathogenesis of extrapulmonary tuberculosis. Diagn Cytopathol 2016; 44: 98-102.

15. Ikram A, Ahmed SA, Khan FA, Khadim MT, Satti L. Rapid Mycobacterium tuberculosis DNA detection on fine needle aspirates from extra pulmonary lymph nodes. J Coll Physicians Surg Pak 2015; 25: 417-421.

16. Tadesse M, Abebe G, Abdissa K et al. GeneXpert MTB/RIF Assay for the diagnosis of tuberculous lymphadenitis on concentrated fine needle aspirates in high tuberculosis burden settings. PLoS One 2015; 10: e0137471.

17. Fukui S, Sekiya N, Takizawa Y et al. Disseminated Mycobacterium abscessus infection following septic arthritis: a case report and review of the literature. Medicine (Baltimore) 2015; 94: e861.

18. Morales P, Gil A, Santos M. Mycobacterium abscessus infection in transplant recipients. Transplant Proc 2010; 42: 3058-3060.

19. Ramagopalan SV, Goldacre R, Skingsley A, Conlon C, Goldacre MJ. Associations between selected immune-mediated diseases and tuberculosis: record-linkage studies. BMC Med 2013; 11: 97. doi: 10.1186/1741-7015-11-97.

20. Airio A, Kauppi M, Kautiainen H, Hakala M, Kinnula V. High association of mycobacterial infections with polymyositis in a non-endemic country for tuberculosis. Ann Rheum Dis 2007; 66: 1404-1405.

21. Wu PH, Lin YT, Yang YH, Lin YC, Lin YC. The increased risk of active tuberculosis disease in patients with dermatomyositis - a nationwide retrospective cohort study. Sci Rep 2015; 5 : 16303. doi: 10.1038/srep16303. 